\title{
On the Extension of the Three-Term Recurrence Relation to Probabilities Distributions without Moments
}

\author{
Habib Rebei, Anis Riahi \\ Department of Mathematics, College of Science, Qassim University, Al-Mulida, Saudi Arabia \\ Email: habib.rebei@ipein.rnu.tn, a1riahi@yahoo.fr
}

How to cite this paper: Rebei, H. and Riahi, A. (2018) On the Extension of the Three-Term Recurrence Relation to Probabilities Distributions without Moments. Journal of Applied Mathematics and Physics, 6, 588-601.

https://doi.org/10.4236/jamp.2018.63051

Received: December 18, 2017

Accepted: February 10, 2018

Published: March 28, 2018

Copyright ( 92018 by authors and Scientific Research Publishing Inc. This work is licensed under the Creative Commons Attribution International License (CC BY 4.0).

http://creativecommons.org/licenses/by/4.0/

\section{Open Access}

\begin{abstract}
In this paper, we extend the three-term recurrence relation for orthogonal polynomials associated with a probability distribution having a finite moment of all orders to a class of orthogonal functions associated with an infinitely divisible probability distribution $\mu$ having a finite moments of order less or equal to four. An explicit expression of these functions will be given in term of the Lévy-Khintchine function of the measure $\mu$.
\end{abstract}

\section{Keywords}

Three-Term Recurrence Relation, Quantum Decomposition of Random Variables Without Moments, Lévy-Khintchine Function

\section{Introduction}

It has been known from [1] and [2] that for every probability distribution $\mu$ with finite moments of all orders, there exits a family of monic orthogonal polynomials $P_{n}$ and a paire of sequences $\alpha_{0}, \alpha_{1}, \cdots$ and $w_{0}=1, w_{1}, w_{2}, \cdots>0$ satisfying the three-term recurrence relation (or the tri-diagonal Jacobi relation)

$$
\begin{gathered}
P_{-1}=0 \\
P_{0}=1 \\
x P_{n}=P_{n+1}+\alpha_{n} P_{n}+w_{n} P_{n-1}, \quad n \in \mathbb{N}:=\{0,1,2, \cdots\}
\end{gathered}
$$

The sequences $\left(\alpha_{n}\right)$ and $\left(w_{n}\right)$ are called the Szego-Jacobi parameters of $\mu$.

The starting point of the quantum probabilistic approach to the theory of orthogonal polynomials (OP) is an operator interpretation of the tri-diagonal Jacobi relation (3) in terms of Creation, Annihilation and Preservation (CAP) 
operators. This allows to associate, in a canonical way, to any random variable with all moments commutation relations that generalize the Heisenberg commutation relations (corresponding to the Gauss-Poisson class). From the mathematical point of view, this approach has led to some new results in the theory of OP.

In order to give this operator interpretation, we shall recall the notion of the interacting Fock probability space associated with the measure $\mu$ (See [3] for more details).

Consider an infinite-dimensional separable Hilbert space $\mathcal{H}$, in which a complete orthonormal basis $\left\{\Phi_{n} ; n \in \mathbb{N}\right\}$ is chosen. Let $\mathcal{H}_{0} \subset \mathcal{H}$ denote the dense subspace spanned by the complete orthonormal basis $\left\{\Phi_{n}\right\}$.

Given the sequence $\left\{w_{n}, n \in \mathbb{N}\right\}$, we associate linear operators $B^{ \pm} \in L\left(\mathcal{H}_{0}\right)$ given by:

$$
\begin{gathered}
B^{+} \Phi_{n}=\sqrt{w_{n+1}} \Phi_{n+1} \\
B^{-} \Phi_{0}=0, \quad B^{-} \Phi_{n}=\sqrt{w_{n}} \Phi_{n-1}, \quad n \geq 1 .
\end{gathered}
$$

Its known that $B^{ \pm}$are mutually adjoint and the linear subspace $\Gamma \subset \mathcal{H}_{0}$ spanned by the set $\left\{\left(B^{+}\right)^{n} \Phi_{0}, n=0,1,2, \cdots\right\}$ is invariant under the action of $B^{ \pm}$.

The quadruple $\left\{\Gamma,\left(\Phi_{n}\right)_{n}, B^{ \pm}\right\}$is called the interacting Fock probability space associated with $\mu$. The operators $B^{+}$and $B^{-}$are called the creation operator and the annihilation operators respectively. The linear operator given by

$$
N \Phi_{n}=n \Phi_{n}, n=0,1,2, \cdots
$$

is called the number operator. More generally, with the sequence $\left\{\alpha_{n} ; n=1,2, \cdots\right\}$, we associate the preservation operator $\alpha_{N} \in L(\Gamma)$ by the prescription

$$
\alpha_{N} \Phi_{n}=\alpha_{n} \Phi_{n}, \quad n=1,2, \cdots
$$

Let $L_{\mathbb{C}}^{2}(\mathbb{R}, \mu)$ be the space of classes of complex valued, square integrable functions w.r.t $\mu$. In the following, we simply denote it by $L^{2}(\mu)$ and we assume that the sub-space $\mathcal{P}(\mathbb{R}, \mu) \subset L^{2}(\mu)$ spanned by the polynomial functions is dense in $L^{2}(\mu)$. So that $\left(P_{n}\right)_{n}$ is an Hilbertian basis of $L^{2}(\mu)$. In such case, we consider the isomorphism $U$ from $\Gamma$ to $L^{2}(\mu)$ whose its restriction on $\mathcal{H}_{0}$ given by:

$$
U: \Phi_{0} \mapsto P_{0}, \quad \sqrt{w_{n}} ! \Phi_{n} \mapsto P_{n}, \quad n \in \mathbb{N}^{*}=\{1,2, \cdots\},
$$

where $w_{n} !=w_{0} w_{1} \cdots w_{n}$. Then the $U$ is unitary and we have

$$
\begin{gathered}
\qquad P_{n}=P_{n+1}+\alpha_{n} P_{n}+w_{n} P_{n-1} \\
\Leftrightarrow q U\left(\sqrt{w_{n} !} \Phi_{n}\right)=U\left(\sqrt{w_{n+1} !} \Phi_{n+1}+\alpha_{n} \sqrt{w_{n} !} \Phi_{n}+w_{n} \sqrt{w_{n-1} !} \Phi_{n-1}\right) \\
\Leftrightarrow \sqrt{w_{n} !} U\left(U^{-1} q U\right) \Phi_{n}=\sqrt{w_{n} !} U\left(\sqrt{w_{n+1}} \Phi_{n+1}+\alpha_{n} \Phi_{n}+\sqrt{w_{n}} \Phi_{n-1}\right) \\
\Leftrightarrow U\left(U^{-1} q U\right) \Phi_{n}=U\left(B^{+} \Phi_{n}+\alpha_{N} \Phi_{n}+B^{-} \Phi_{n}\right)
\end{gathered}
$$




$$
\begin{aligned}
& \Leftrightarrow\left(U^{-1} q U\right) \Phi_{n}=\left(B^{+}+\alpha_{N}+B^{-}\right) \Phi_{n} \\
& \Leftrightarrow U^{-1} q U=B^{+}+\alpha_{N}+B^{-}
\end{aligned}
$$

This means that the field operator $T:=B^{+}+\alpha_{N}+B^{-}$is the $U^{-1}$-image of the position operator $q:=M_{x}$ on $L^{2}(\mu)$ providing, in this way, a new interpretation of the recursion relation driving by OP in term of CAP operators. Since the random variable with distribution $\mu$ can be identified, up to stochastic equivalence $\equiv$, with the position operator $q$ on $L^{2}(\mu)$, the previous new formulation of the tri-diagonal Jacobi relation in term of the CAP operators is called the quantum decomposition of the classical random variable. In fact we have seen that

$$
q \equiv T=B^{+}+\alpha_{N}+B^{-}
$$

This shows that any classical random variable has a built in non commutative structure which is intrinsic and canonical, and not artificially put by hands, that is a sum of three non commuting random variables.

This result motivated the apparition of a series of papers [4]-[9] dealing in the same context and provided many applications in the theory of quantum probability. In the paper [4], a similar result was obtained but for the family of random variables having an infinitely divisible distribution (I.D-distribution in the following) and having only the moment of the second order. Here, similarity means that the quantum decomposition can be obtained also for this family of random variables.

Based on the notion of the positive definite kernel and using the Lévy-Khintchine function established for the I.D-distributions, the paper [4] constructed a natural isomorphism $U$ from the Fock space $\Gamma\left(L^{2}(v)\right)$ over the $L^{2}$-space w.r.t the Lévy measure $v$ to the space $L^{2}(\mu)$. Then the $U^{-1}$-image of the position operator $q$ is the field operator

$$
Q=A^{+}(\mathfrak{q})+A^{-}(\mathfrak{q})+\Lambda(q)+E(X) \mathbf{1}
$$

where $\mathfrak{q}$ is the function $(t \mapsto \mathfrak{q}(t):=(q \cdot 1)(t)=t) \in L^{2}(v)$. See papers [10] and [11] in which the operator $Q$ was widely studied.

In this approach, the construction was not based on the orthogonal polynomials sequence associated with $\mu$. But it required only the infinite divisibility property, where the Lévy-Khinchine function have played an important role. Then one can ask about the analytic form of the relation (4), or equivalently the counterpart of the three-term recurrence relation. The only obscure point is the existence of such an analogue of the sequence of the orthogonal polynomials. Since the hypothesis on moments is not satisfied, such a sequence of orthogonal polynomial does exist. But the isomorphism $U$ provided us a such chaos-decomposition of the space $L^{2}(\mu)$. For this reason we ask the question if there exist a such analogue for the family of orthogonal polynomial, if it is the case it must be a total family of orthogonal functions in the space $L^{2}(\mu)$ satisfying a recursion relation similar to the well known for OP. 
This paper is organized as follows:

In Section 2, we recall some known facts about the bosonic Fock space and the quantum decomposition of classical random variables without moments, having I.D-distributions, obtained in [12] [4] and [5]. In Section 3, we compute the action of the generalized field operator $Q=U^{-1} q U$ on the $n^{\text {th }}$ particle vectors $\left(Q_{n, \alpha}\right)$. The main result of this paper will be given in Section 4, so that we compute the action of the position operator $q=M_{x}$ on the orthogonal functions $E_{n, \alpha}$. This provide such a generalization of the tri-diagonal recursion relation for OP. Finally, the explicit form of theses functions will be given.

\section{Preliminaries}

\subsection{The Bosonic Fock Space}

Let $\mathcal{H}$ be a separable Hilbert space. Let us denote $\mathcal{H}^{\otimes n}$ (resp. $f^{\otimes n}$ ) the tensor product of $n$-copies of $\mathcal{H}$ (resp. $f \in \mathcal{H}$ ) and let $u_{\sigma}$ be the unique unitary operator such that

$$
u_{\sigma}: \mathcal{H}^{\otimes n} \rightarrow \mathcal{H}^{\otimes n}, \quad f_{1} \otimes \cdots \otimes f_{n} \mapsto f_{\sigma(1)} \otimes \cdots \otimes f_{\sigma(n)}
$$

where $\sigma \in \mathfrak{S}_{n}$ is a permutation of $n$-variables.

Let $\mathcal{H}^{\otimes 0}:=\mathbb{C} \Phi$, were $\Phi$ is the vacuum vector, let

$$
S_{n}:=\frac{1}{n !} \sum_{\sigma \in \mathfrak{S}_{n}} u_{\sigma}: \mathcal{H}^{\otimes n} \rightarrow \mathcal{H}^{\odot n}
$$

be the orthogonal projection.

We define

$$
\Gamma(\mathcal{H}):=\bigoplus_{n=0}^{\infty} \mathcal{H}^{\odot n}
$$

where $\mathcal{H}^{\odot n}=S_{n}\left(\mathcal{H}^{\otimes n}\right)$.

Let us denote

$$
\operatorname{Exp}(f):=\sum_{n=0}^{\infty} \frac{f^{\otimes n}}{\sqrt{n !}}, \quad f \in \mathcal{H} .
$$

Then $\langle\operatorname{Exp}(f), \operatorname{Exp}(g)\rangle=e^{\langle f, g\rangle}$. Moreover, the set $\{\operatorname{Exp}(f): f \in \mathcal{H}\}$ is linearly independent dense in $\Gamma(\mathcal{H})$.

The bosonic creation and annihilation operators are defined, on the total set

$$
\left\{S_{n}\left(v_{1} \otimes \cdots \otimes v_{n}\right) \in \mathcal{H}^{\odot n}: v_{1}, \cdots, v_{n} \in \mathcal{H}\right\}
$$

as follows:

For $u \in \mathcal{H}$,

$$
\begin{gathered}
A^{+}(u): \mathcal{H}^{\odot n} \rightarrow \mathcal{H}^{\odot(n+1)} \\
S_{n}\left(v_{1} \otimes \cdots \otimes v_{n}\right) \mapsto \sqrt{n+1} S_{n+1}\left(u \otimes v_{1} \otimes \cdots \otimes v_{n}\right),
\end{gathered}
$$

and

$$
A^{+}(u) \Phi=u
$$




$$
\begin{gathered}
A^{-}(u): \mathcal{H}^{\odot n} \rightarrow \mathcal{H}^{\odot(n-1)} \\
S_{n}\left(v_{1} \otimes \cdots \otimes v_{n}\right) \mapsto \frac{1}{\sqrt{n}} \sum_{i=1}^{n}\left\langle u, v_{i}\right\rangle S_{n-1}\left(v_{1} \otimes \cdots \otimes \widehat{v_{i}} \otimes \cdots \otimes v_{n}\right),
\end{gathered}
$$

and

$$
A^{-}(u) \Phi=0,
$$

where $\hat{x}$ denotes omission of the corresponding variable. The preservation operator associated with the self adjoint operator $T$ on $\mathcal{H}$ is given by:

$$
\Lambda(T): S_{n}\left(v_{1} \otimes \cdots \otimes v_{n}\right) \in \mathcal{H}^{\odot n} \mapsto \sum_{i=1}^{n} S_{n}\left(v_{1} \otimes \cdots \otimes T v_{i} \otimes \cdots \otimes v_{n}\right) \in \mathcal{H}^{\odot n}
$$

\subsection{The Quantum Decomposition of Classical Random Variables with I.D-Distributions}

In this section, we recall briefly, what has been obtained in the paper [4] around quantum decomposition of random variables with I.D-distributions and having a finite second order moment.

Let us consider a random variable $X$ with I.D-probability distribution $\mu$ having a finite second order moment. It is known (see [13]), that the Fourier transform of $\mu$ given by

$$
\hat{\mu}(x)=\mathrm{e}^{\Psi(x)} ; \quad x \in \mathbb{R},
$$

where $\Psi$ is given by

$$
\Psi(x)=i \gamma x-\frac{\sigma^{2}}{2} x^{2}+\int_{\mathbb{R}\{00\}}\left(\mathrm{e}^{i x t}-1-\frac{i x t}{1+t^{2}}\right) v(\mathrm{~d} t) ; \quad x \in \mathbb{R}
$$

such that $\gamma, \sigma \in \mathbb{R}$ and $\nu$ is the the Lévy measure of $\mu$. The function $\Psi$ is called the Lévy-Khintchine function or the characteristic exponent associated with $\mu$.

Since the second order moment of $\mu$ is finite, the same result will be true for $v$, i.e,

$$
\int_{\mathbb{R}\{\{0\}}|t|^{2} v(\mathrm{~d} t)<+\infty .
$$

We suppose also that the gaussian part of $\mu$ is null (i.e., $\sigma=0$ ). Under these conditions, we have the following results:

The family $\left\{e_{x}: \mathbb{R} \rightarrow \mathbb{C}, t \mapsto \mathrm{e}^{i t x}\right\}$ of the trigonometric functions is total in $L^{2}(\mu)$ and the family of the functions

$$
f_{x}(t):=\mathrm{e}^{i x t}-1, \quad x \in \mathbb{R}
$$

is total in $L^{2}(v)$.

Then by applying the Araki-Woods-Parthasarathy-Schmidt isomorphism in [12] for the infinitely divisible positive definite kernel

$$
k(x, y):=\mathrm{e}^{\Psi(y-x)}, \quad x, y \in \mathbb{R},
$$

we have proved the following theorem (See [4] for more details and descriptions).

Theorem 2.1. The unique linear operator $U$ given on the exponential vectors 
$\left\{\operatorname{Exp}\left(f_{x}\right), x \in \mathbb{R}\right\}$ by

$$
\begin{gathered}
U: \Gamma\left(L^{2}(v)\right) \rightarrow L^{2}(\mu) \\
\operatorname{Exp}\left(f_{x}\right) \mapsto U\left(\operatorname{Exp}\left(f_{x}\right)\right):=\mathrm{e}^{-\psi(x)} e_{x}
\end{gathered}
$$

is an unitary isomorphism from the Fock space $\Gamma\left(L^{2}(v)\right)$ to $L^{2}(\mu)$.

Definition 1. Let $q$ be the multiplication (position) operator in $L^{2}(\mu)$ :

$$
(q f)(t):=t f(t) ; \quad f \in L^{2}(\mu), t \in \mathbb{R}
$$

Define the operator $Q$ on $\Gamma\left(L^{2}(v)\right)$ by

$$
Q:=U^{*} q U
$$

where $U$ is the isomorphism defined by (12). Since $\mu$ is a finite measure on $\mathbb{R}$, the operator $q$ is self-adjoint (see [14] Proposition 1, chapter VIII. 3) and

$$
\mathrm{e}^{i t Q}=U^{*} \mathrm{e}^{i t q} U, \quad t \in \mathbb{R}
$$

The operator $Q$ is called the generalized field operator.

It follows from condition (10) that the total set $\left\{\operatorname{Exp}\left(f_{y}\right), y \in \mathbb{R}\right\}$ is in the domain of $Q$. Moreover, one has the following theorem:

Theorem 2.2. Let $\mathfrak{q}$ be the function given by

$$
\mathfrak{q}(t)=t, \quad t \in \mathbb{R} .
$$

Then the generalized field operator $Q$ takes the form

$$
Q=A_{v}^{+}(\mathfrak{q})+A_{v}^{-}(\mathfrak{q})+\Lambda_{v}(q)+m \mathbf{1},
$$

where $m=E(X)$, the expectation of $X$, and $A_{v}^{+}, A_{v}^{-}, \Lambda_{v}$ are the creation, annihilation and preservation operators in the Fock space $\Gamma\left(L^{2}(v)\right)$ given by the prescriptions as in (5)-(7).

\section{The Generalized Field Operator}

\subsection{Notations}

We denote by $\tau$ the set of all sequences of non negatives integers with finite number of nonzero entries. In the sequel $\mathbb{N}^{n}$ (resp. $\mathbb{C}^{n}$ ) will be interpreted as subset of the set $\tau$ (resp. $l^{2}(\mathbb{C})$ ). Throughout the remain of this paper we shall use the following notations:

For $z=\left(z_{1}, \cdots, z_{n}, z_{n+1}, \cdots\right) \in l^{2}(\mathbb{C}), \alpha=\left(\alpha_{1}, \cdots, \alpha_{n}, 0,0, \cdots\right)$ and $\beta=\left(\beta_{1}, \cdots, \beta_{n}, 0,0, \cdots\right) \in \tau$,

$$
\begin{gathered}
\|z\|^{2}=\sum_{k=1}^{\infty}\left|z_{k}\right|^{2} ; \quad z^{\alpha}=z_{1}^{\alpha_{1}} \cdots z_{n}^{\alpha_{n}} ; \quad \alpha !=\alpha_{1} ! \cdots \alpha_{n} ! \\
\alpha+\beta=\left(\alpha_{1}+\beta_{1}, \cdots, \alpha_{n}+\beta_{n}, \cdots\right) ; \quad|\alpha|=\sum_{k=1}^{\infty} \alpha_{k} ; \quad e_{\alpha}=\frac{z^{\alpha}}{\sqrt{\alpha !}}
\end{gathered}
$$

The support of such element $\alpha \in \tau$ is defined by

$$
\pi(\alpha):=\left\{j \in \mathbb{N}^{*}: \alpha_{j} \neq 0\right\}
$$

When $g=\left(g_{1}, \cdots, g_{m}, g_{m+1}, \cdots\right)$ is a sequence of elements of an Hilbert space 
$\mathcal{H}$ and $\alpha=\left(\alpha_{1}, \cdots, \alpha_{m}, 0,0, \cdots\right) \in \tau$, we denote

$$
g^{\otimes \alpha}:=g_{1}^{\otimes \alpha_{1}} \otimes \cdots \otimes g_{m}^{\otimes \alpha_{m}} \in \mathcal{H}^{\otimes|\alpha|}
$$

In particular if $\pi(\alpha)=i_{1}, \cdots, i_{s}$ and $|\alpha|=n$, so that $\alpha$ takes the form

$$
\alpha=\left(0, \cdots, 0, \alpha_{i_{1}}, 0, \cdots, 0, \alpha_{i_{2}}, 0, \cdots, 0, \alpha_{i_{s}}, 0,0, \cdots\right) \quad \text { with } \alpha_{i_{1}}+\cdots+\alpha_{i_{s}}=n,
$$

then

$$
g^{\otimes \alpha}=\bigotimes_{j \in \pi(\alpha)} g_{j}^{\otimes \alpha_{j}}=\bigotimes_{j=1}^{s} g_{i_{j}}^{\otimes \alpha_{i j}}
$$

From [15], we recall the following identity which is the analogue of the multinomial Newton formula

$$
\left(\sum_{k=1}^{\infty} g_{k}\right)^{\otimes n}=\sum_{\alpha \in \tau,|\alpha|=n} \frac{n !}{\alpha !} S_{n}\left(g^{\otimes \alpha}\right),
$$

which take place whenever the series $\sum_{j=1}^{\infty} g_{j}$ is convergent.

If $\mathcal{H}$ is separable and $\left\{g_{k}\right\}_{k \geq 1}$ is an Hilbertian basis of it, then the set

$$
\left\{Q_{n}(\alpha):=\sqrt{\frac{n !}{\alpha !}} S_{n}\left(g^{\otimes \alpha}\right):|\alpha|=n, n \in \mathcal{H}\right\}
$$

is an orthonormal basis of the Hilbert space $\mathcal{H}^{\odot n}$, with the convention $Q_{0}=\Phi$.

Let $\left\{\delta_{j}, j=1,2, \cdots\right\}$ be the canonic basis of $l^{2}(\mathbb{C})$. For $\alpha \in \tau$, we denote

$$
\alpha+k:=\alpha+\delta_{k}=\left(\alpha_{1}, \alpha_{2}, \cdots, \alpha_{k-1}, \alpha_{k}+1, \alpha_{k+1}, \cdots\right), \quad k=1,2, \cdots
$$

and

$$
\alpha-k:=\alpha-\delta_{k}=\left(\alpha_{1}, \alpha_{2}, \cdots, \alpha_{k-1}, \alpha_{k}-1, \alpha_{k+1}, \cdots\right), \quad k \in \pi(\alpha) \subset \mathbb{N}^{*} .
$$

Note that if $k \notin \pi(\alpha)$, then $\alpha-k$ can be defined as in (16), however it is not an element of $\tau$, because its $k^{\text {th }}$-entry $(\alpha-k)_{k}=-1$. In this case, we adapt by convention that

$$
Q_{n}(\alpha-k)=0 .
$$

Finally, we recall that

$$
\begin{gathered}
(\alpha+k) !=\left(\alpha_{k}+1\right) \alpha !, k \in \mathbb{N}^{*} \\
(\alpha-k) !=\frac{\alpha !}{\alpha_{k}}, k \in \pi(\alpha)
\end{gathered}
$$

\subsection{Computation of the Action of the Generalized Field Operator on the Basis $\left(\Phi_{n}\right)_{n}$}

In the remain, we take $\mathcal{H}=L^{2}(v)$ and we assume that second order moment of $v$ is finite. Let $g_{1}$ be the function given by $g_{1}(t):=\frac{\mathfrak{q}(t)}{v(\mathfrak{q})}$, where

$$
v(\mathfrak{q}):=\left(\int_{\mathbb{R}} t^{2} v(\mathrm{~d} t)\right)^{\frac{1}{2}} .
$$

Since the set $\left\{f_{x}, x \in \mathbb{R}\right\}$ is total in $L^{2}(v)$ (See (11)), then 
$\mathcal{E}:=\left\{g_{1}\right\} \cup\left\{f_{x}, x \in \mathbb{Q}\right\}$ is also total. Then by the Gram-Schmidt procedure, we construct an Hilbertian basis of it, that is denoted by

$$
g=\left(g_{1}, g_{2}, \cdots, g_{k}, \cdots\right)
$$

Lemma 3.1. If the $4^{\text {th }}$-moment of $v$ is finite then $g_{k} \in \operatorname{Dom}(q) \subset L^{2}(v)$ for all $k=1,2, \ldots$.

Proof. We have

$$
\int_{\mathbb{R}}\left|\left(q g_{1}\right)(t)\right|^{2} v(\mathrm{~d} t)=\int_{\mathbb{R}}\left|\frac{t^{2}}{v(\mathfrak{q})}\right|^{2} v(\mathrm{~d} t)=\frac{1}{(v(\mathfrak{q}))^{2}} \int_{\mathbb{R}} t^{4} v(\mathrm{~d} t)<+\infty
$$

Then $g_{1} \in \operatorname{Dom}(q)$. Since $g_{k} \in \operatorname{Span}\left\{g_{1}, f_{x_{j}}, j=1,2, \cdots\right\}$ for all $k=2,3, \cdots$, then it is sufficient to prove that $f_{x} \in \operatorname{Dom}(q)$.

We have

$$
\int_{\mathbb{R}}\left|\left(q f_{x}\right)(t)\right|^{2} v(\mathrm{~d} t)=\int_{\mathbb{R}} t^{2}\left|\mathrm{e}^{i x t}-1\right|^{2} v(\mathrm{~d} t) \leq \int_{\mathbb{R}} 4 t^{2} v(\mathrm{~d} t)<+\infty,
$$

where we have used the condition (10).

$$
q g_{j}=\sum_{k=1}^{\infty}\left\langle g_{k}, q g_{j}\right\rangle g_{k}
$$

Proposition 3.1. Let $\left\{R_{n}(\alpha), n \in \mathbb{N}, \alpha \in \tau:|\alpha|=n, n \in \mathbb{N}\right\}$ be the orthogonal basis of $\Gamma\left(L^{2}(v)\right)$ given by

$$
R_{n}(\alpha):=\sqrt{\frac{n !}{\alpha !}} Q_{n}(\alpha)=\frac{n !}{\alpha !} S_{n}\left(g^{\otimes \alpha}\right) ; \quad R_{0}:=\Phi,
$$

where $g=\left(g_{1}, g_{2}, \cdots, g_{k}, \cdots\right)$ is the basis given by (20). Then we have

$$
\begin{gathered}
A^{+}(\mathfrak{q}) R_{n}(\alpha)=\frac{v(\mathfrak{q})\left(\alpha_{1}+1\right)}{\sqrt{n+1}} R_{n+1}(\alpha+1) \\
A^{-}(\mathfrak{q}) R_{n}(\alpha)=v(\mathfrak{q}) \sqrt{n} R_{n-1}(\alpha-1) \\
\Lambda(q) R_{n}(\alpha)=s_{\alpha} R_{n}(\alpha)+\sum_{j \in \pi(\alpha)} \sum_{k \geq 1, k \neq j}^{\infty}\left(\alpha_{k}+1\right)\left\langle g_{k}, q g_{j}\right\rangle R_{n}(\alpha-j+k)
\end{gathered}
$$

where $s_{\alpha}=\sum_{j \in \pi(\alpha)} \alpha_{j}\left\langle g_{j}, q g_{j}\right\rangle$.

Remark 1. Note that the relation (22) still true in the case when $1 \notin \pi(\alpha)$ with convention that $R_{n-1}(\alpha-1)=0$.

Proof. From (5), we have

$$
\begin{aligned}
A^{+}(\mathfrak{q}) R_{n}(\alpha) & =A^{+}\left(v(\mathfrak{q}) g_{1}\right) R_{n}(\alpha) \\
& =v(\mathfrak{q}) A^{+}\left(g_{1}\right) R_{n}(\alpha) \\
& =v(\mathfrak{q}) \frac{n !}{\alpha !} A^{+}\left(g_{1}\right) S_{n}\left(g^{\otimes \alpha}\right) \\
& =v(\mathfrak{q}) \frac{n ! \sqrt{n+1}}{\alpha !} S_{n+1}\left(g_{1} \otimes g^{\otimes \alpha}\right) \\
& =v(\mathfrak{q}) \frac{n ! \sqrt{n+1}}{\alpha !} S_{n+1}\left(g^{\otimes(\alpha+1)}\right)
\end{aligned}
$$




$$
\begin{aligned}
& =v(\mathfrak{q}) \frac{n ! \sqrt{n+1}}{\alpha !} \frac{(\alpha+1) !}{(n+1) !} \frac{(n+1) !}{(\alpha+1) !} S_{n+1}\left(g^{\otimes(\alpha+1)}\right) \\
& =v(\mathfrak{q}) \frac{n ! \sqrt{n+1}}{\alpha !} \frac{(\alpha+1) !}{(n+1) !} R_{n+1}(\alpha+1) \\
& =v(\mathfrak{q}) \frac{n ! \sqrt{n+1}}{\alpha !} \frac{\left(\alpha_{1}+1\right) \alpha !}{(n+1) !} R_{n+1}(\alpha+1) \\
& =\frac{v(\mathfrak{q})\left(\alpha_{1}+1\right)}{\sqrt{n+1}} R_{n+1}(\alpha+1) .
\end{aligned}
$$

This prove (21).

From (5), we have

$$
\begin{aligned}
A^{-}(\mathfrak{q}) R_{n}(\alpha) & =A^{-}\left(v(\mathfrak{q}) g_{1}\right) R_{n}(\alpha) \\
& =v(\mathfrak{q}) A^{-}\left(g_{1}\right) R_{n}(\alpha) \\
& =v(\mathfrak{q}) \frac{n !}{\alpha !} A^{-}\left(g_{1}\right) S_{n}\left(g^{\otimes \alpha}\right) \\
& =v(\mathfrak{q}) \frac{n !}{\alpha !} \frac{1}{\sqrt{n}} \sum_{j \in \pi(\alpha)} \alpha_{j}\left\langle g_{1}, g_{j}\right\rangle S_{n-1}\left(g^{\otimes(\alpha-j)}\right)
\end{aligned}
$$

Here, we have two cases:

If $1 \in \pi(\alpha)$, then (24), becomes

$$
\begin{aligned}
A^{-}(\mathfrak{q}) R_{n}(\alpha) & =v(\mathfrak{q}) \frac{n !}{\alpha !} \frac{1}{\sqrt{n}} \alpha_{1}\left\langle g_{1}, g_{1}\right\rangle S_{n-1}\left(g^{\otimes(\alpha-1)}\right) \\
& =v(\mathfrak{q}) \frac{n !}{\alpha !} \frac{1}{\sqrt{n}} \alpha_{1} \frac{(\alpha-1) !}{(n-1) !} R_{n-1}(\alpha-1) \\
& =v(\mathfrak{q}) \sqrt{n} R_{n-1}(\alpha-1)
\end{aligned}
$$

If $1 \notin \pi(\alpha)$, then $\left\langle g_{1}, g_{j}\right\rangle=0$ for all $j \in \pi(\alpha)$. Therefore (24) gives

$$
A^{-}(\mathfrak{q}) R_{n}(\alpha)=0 .
$$

But in view of (17), we have $R_{n-1}(\alpha-1)=0$ which gives that the relation (25) sill true. Hence (22) is proved.

Now, it remains to justify (23). From (7), we get

$$
\Lambda(q) R_{n}(\alpha)=\frac{n !}{\alpha !} \sum_{j \in \pi(\alpha)} \alpha_{j} S_{n}\left(q g_{j} \otimes g^{\otimes(\alpha-j)}\right) .
$$

Since $q g_{j} \in L^{2}(v)$, then it can be written as follows:

$$
q g_{j}=\sum_{k=1}^{\infty}\left\langle g_{k}, q g_{j}\right\rangle g_{k}
$$

Using the fact that $S_{n}$ is bounded, the Equation (26) becomes

$$
\begin{aligned}
\Lambda(q) R_{n}(\alpha) & =\frac{n !}{\alpha !} \sum_{j \in \pi(\alpha)} \alpha_{j} \sum_{k=1}^{\infty}\left\langle g_{k}, q g_{j}\right\rangle S_{n}\left(g_{k} \otimes g^{\otimes(\alpha-j)}\right) \\
& =\sum_{j \in \pi(\alpha)} \sum_{k=1}^{\infty}\left\langle g_{k}, q g_{j}\right\rangle \frac{\alpha_{j}(\alpha-j+k) !}{\alpha !} \frac{n !}{(\alpha-j+k) !} S_{n}\left(g_{k} \otimes g^{\otimes(\alpha-j)}\right) \\
& =\sum_{j \in \pi(\alpha)} \sum_{k=1}^{\infty}(\alpha-j+k) ! \frac{\alpha}{\alpha !}\left\langle g_{k}, q g_{j}\right\rangle R_{n}(\alpha-j+k)
\end{aligned}
$$


But we have for $j \in \pi(\alpha)$,

$$
(\alpha-j+k) != \begin{cases}\frac{\left(\alpha_{k}+1\right) \alpha !}{\alpha_{j}}, & k \neq j \\ \alpha !, & k=j .\end{cases}
$$

Then (27) becomes

$$
\begin{aligned}
\Lambda(q) R_{n}(\alpha)= & \sum_{j \in \pi(\alpha)} \sum_{k \geq 1, k \neq j} \frac{\left(\alpha_{k}+1\right) \alpha !}{\alpha_{j}} \frac{\alpha_{j}}{\alpha !}\left\langle g_{k}, q g_{j}\right\rangle R_{n}(\alpha-j+k) \\
& +\sum_{j \in \pi(\alpha)} \alpha ! \frac{\alpha_{j}}{\alpha !}\left\langle g_{j}, q g_{j}\right\rangle R_{n}(\alpha) \\
= & \sum_{j \in \pi(\alpha)} \sum_{k \geq 1, k \neq j}\left(\alpha_{k}+1\right)\left\langle g_{k}, q g_{j}\right\rangle R_{n}(\alpha-j+k) \\
& +\left(\sum_{j \in \pi(\alpha)} \alpha_{j}\left\langle g_{j}, q g_{j}\right\rangle\right) R_{n}(\alpha)
\end{aligned}
$$

This ends the proof.

Corollary 3.1.1 The action of the generalized field operator $Q$ on the basis $\left\{R_{n}(\alpha) ; n, \alpha\right\}$ is given as follows.

$$
\begin{aligned}
Q R_{n}(\alpha)= & \frac{v(\mathfrak{q})\left(\alpha_{1}+1\right)}{\sqrt{n+1}} R_{n+1}(\alpha+1)+\left(m+s_{\alpha}\right) R_{n}(\alpha)+v(\mathfrak{q}) \sqrt{n} R_{n-1}(\alpha-1) \\
& +\sum_{j \in \pi(\alpha)} \sum_{k \geq 1, k \neq j}\left(\alpha_{k}+1\right)\left\langle g_{k}, q g_{j}\right\rangle R_{n}(\alpha-j+k)
\end{aligned}
$$

Proof. A straightforward computations.

\section{Orthogonal Functions and Generalization of the Three-Term Recurrence Relation}

In this section, we give the action of the multiplication operator $q$ on the functions

$$
E_{n, \alpha}:=U\left(R_{n}(\alpha)\right), n \in \mathbb{N}, \alpha \in \tau,|\alpha|=n \in \mathbb{N} .
$$

Then we deduce the generalization of the three-term recurrence relation in term of the orthogonal functions $E_{n, \alpha}$.

Since $U$ is unitary from $\Gamma\left(L^{2}(v)\right)$ to $L^{2}(\mu)$ and $\left\{R_{n}(\alpha)\right\}$ is an orthogonal basis of $\Gamma\left(L^{2}(v)\right)$, the family $\left\{E_{n, \alpha}=U\left(R_{n}(\alpha)\right), n, \alpha\right\}$ is an orthogonal basis of $L^{2}(\mu)$.

Theorem 4.1 Let $L_{n}^{2}(\mu):=U\left(L^{2}(v)^{\oplus n}\right)$ and let $D_{n}$ be the diagonal operator from $L_{n}^{2}(\mu)$ to itself given by

$$
D_{n}\left(E_{n, \alpha}\right):=\sum_{j \in \pi(\alpha)} \sum_{k \geq 1, k \neq j}\left(\alpha_{k}+1\right)\left\langle g_{k}, q g_{j}\right\rangle E_{n, \alpha-j+k} .
$$

Then for all $n \in \mathbb{N}, \alpha \in \tau,|\alpha|=n$, we have

$$
q E_{n, \alpha}=\frac{v(\mathfrak{q})\left(\alpha_{1}+1\right)}{\sqrt{n+1}} E_{n+1, \alpha+1}+\left(m+s_{\alpha}\right) E_{n, \alpha}+v(\mathfrak{q}) \sqrt{n} E_{n-1, \alpha-1}+D_{n}\left(E_{n, \alpha}\right)
$$

Remark 2. Since $U$ is unitary and the basis $\left\{R_{n}(\alpha), n \in \mathbb{N}, \alpha \in \tau,|\alpha|=n\right\}$ is 
orthogonal, then $\left\{E_{n, \alpha}=U\left(R_{n}(\alpha)\right), n \in \mathbb{N}^{*}, \alpha \in \tau,|\alpha|=n\right\} \quad$ is an orthogonal basis of $L^{2}(\mu)$. Moreover, the chaos decomposition of the Fock space $\Gamma\left(L^{2}(v)\right)$ induces the following chaos-decomposition of the space $L^{2}(\mu)$

$$
L^{2}(\mu)=\bigoplus_{n=0}^{\infty} L_{n}^{2}(\mu) .
$$

Now comparing the relation (30) with (3), the only difference is the apparition of a corrective expression $D_{n}\left(E_{n, \alpha}\right)$ in (30) which is in the $n^{\text {th }}$ chaos. In the case when it is null, (30) will be exactly the well-known tri-diagonal recurrence relation (3). In this sense the relation (30) can be interpreted as a generalization of the three term recurrence relation. Here, the monic orthogonal polynomial sequence is replaced by a double-entries sequence of orthogonal functions parameterized by $n \in \mathbb{N}$ and $\alpha \in \tau$. In addition to the infinite divisibility property, this generalization require only the existence of the second and fourth order moments of $\mu$.

Proof. From relation (29), we deduce that

$$
\begin{aligned}
q E_{n, \alpha}= & q U\left(R_{n}(\alpha)\right)=U\left(U^{*} q U\right)\left(R_{n}(\alpha)\right)=U Q_{C P} R_{n}(\alpha) \\
= & U\left(\frac{v(\mathfrak{q})\left(\alpha_{1}+1\right)}{\sqrt{n+1}} R_{n+1}(\alpha+1)+\left(m+s_{\alpha}\right) R_{n}(\alpha)+v(\mathfrak{q}) \sqrt{n} R_{n-1}(\alpha-1)\right. \\
& \left.+\sum_{j \in \pi(\alpha)} \sum_{k \geq 1, k \neq j}\left(\alpha_{k}+1\right)\left\langle g_{k}, q g_{j}\right\rangle R_{n}(\alpha-j+k)\right) \\
= & \frac{v(\mathfrak{q})\left(\alpha_{1}+1\right)}{\sqrt{n+1}} U\left(R_{n+1}(\alpha+1)\right)+\left(m+s_{\alpha}\right) U\left(R_{n}(\alpha)\right) \\
& +v(\mathfrak{q}) \sqrt{n} U\left(R_{n-1}(\alpha-1)\right)+\sum_{j \in \pi(\alpha)} \sum_{k \geq 1, k \neq j}\left(\alpha_{k}+1\right)\left\langle g_{k}, q g_{j}\right\rangle U\left(R_{n}(\alpha-j+k)\right) \\
= & \frac{v(\mathfrak{q})\left(\alpha_{1}+1\right)}{\sqrt{n+1}} E_{n+1, \alpha+1}+\left(m+s_{\alpha}\right) E_{n, \alpha}+v(\mathfrak{q}) \sqrt{n} E_{n-1, \alpha-1} \\
& +\sum_{j \in \pi(\alpha)} \sum_{k \geq 1, k \neq j}\left(\alpha_{k}+1\right)\left\langle g_{k}, q g_{j}\right\rangle E_{n, \alpha-j+k} \\
= & \frac{v(\mathfrak{q})\left(\alpha_{1}+1\right)}{\sqrt{n+1}} E_{n+1, \alpha+1}+\left(m+s_{\alpha}\right) E_{n, \alpha}+v(\mathfrak{q}) \sqrt{n} E_{n-1, \alpha-1}+D_{n}\left(E_{n, \alpha}\right)
\end{aligned}
$$

Proposition 4.2. We assume that $\mu$ is continuous w.r.t the Lebesgue measure with Radon-Nikodym derivative $\rho$. Then for all $n \in \mathbb{N}$ and $\alpha \in \tau$, one has

$$
E_{n, \alpha}=\frac{\sqrt{n !}}{\alpha ! \rho} \overline{\mathcal{F}^{-1}\left(\mathrm{e}^{\psi(\cdot)}(v(\cdot))^{\alpha}\right)}
$$

where,

$$
v(x):=\left(\left\langle g_{1}, f_{x}\right\rangle,\left\langle g_{2}, f_{x}\right\rangle,\left\langle g_{3}, f_{x}\right\rangle, \cdots\right) \in l^{2}(\mathbb{C})
$$

Proof. Since $\left\{g_{k}, k \in \mathbb{N}^{*}\right\}$ is an Hilbertian basis of $L^{2}(v)$ and $f_{x} \in L^{2}(v)$,

$$
f_{x}=\sum_{k=1}^{\infty}\left\langle g_{k}, f_{x}\right\rangle g_{k}, \quad x \in \mathbb{R}
$$


where the series converge in $L^{2}(v)$. It follows, from the multinomial Newton formula (14), that

$$
\begin{aligned}
f_{x}^{\otimes n} & =\left(\sum_{k=1}^{\infty}\left\langle g_{k}, f_{x}\right\rangle g_{k}\right)^{\otimes n} \\
& =\sum_{\alpha \in \tau,|\alpha|=n} \frac{n !}{\alpha !} S_{n}\left((v(x))^{\alpha} g^{\otimes \alpha}\right) \\
& =\sum_{\alpha \in \tau,|\alpha|=n}(v(x))^{\alpha} \frac{n !}{\alpha !} S_{n}\left(g^{\otimes \alpha}\right) \\
& =\sum_{\alpha \in \tau,|\alpha|=n}(v(x))^{\alpha} R_{n}(\alpha),
\end{aligned}
$$

where

$$
v(x):=\left(v_{1}, v_{2}, \cdots\right)=\left(\left\langle g_{1}, f_{x}\right\rangle,\left\langle g_{2}, f_{x}\right\rangle,\left\langle g_{3}, f_{x}\right\rangle, \cdots\right) \in l^{2}(\mathbb{C})
$$

This implies that

$$
\operatorname{Exp}\left(f_{x}\right)=\sum_{n=0}^{\infty} \frac{1}{\sqrt{n !}} f_{x}^{\otimes n}=\sum_{n=0}^{\infty} \sum_{\alpha \in \tau,|\alpha|=n} \frac{(v(x))^{\alpha}}{\sqrt{n !}} R_{n}(\alpha)
$$

From the definition of $U$, we get

$$
\begin{aligned}
e_{x} & =\mathrm{e}^{\psi(x)} U\left(\operatorname{Exp}\left(f_{x}\right)\right) \\
& =\mathrm{e}^{\psi(x)} \sum_{n=0}^{\infty} \sum_{\alpha \in \tau,|\alpha|=n} \frac{(v(x))^{\alpha}}{\sqrt{n !}} U\left(R_{n}(\alpha)\right) \\
& =\mathrm{e}^{\psi(x)} \sum_{n=0}^{\infty} \sum_{\alpha \in \tau,|\alpha|=n} \frac{(v(x))^{\alpha}}{\sqrt{n !}} E_{n, \alpha},
\end{aligned}
$$

which is the decomposition of $e_{x}$ in the basis $\left\{E_{n, \alpha}, n \in \mathbb{N}, \alpha \in \tau\right\}$. Then

$$
\begin{aligned}
\left\langle E_{n, \alpha}, e_{x}\right\rangle & =\left\langle E_{n, \alpha}, \mathrm{e}^{\psi(x)} \sum_{m=0}^{+\infty} \sum_{\beta \in \tau,|\beta|=m} \frac{(v(x))^{\beta}}{\sqrt{m !}} E_{m, \beta}\right\rangle \\
& =\mathrm{e}^{\psi(x)} \frac{(v(x))^{\alpha}}{\sqrt{n !}}\left\|E_{n, \alpha}\right\|^{2} \\
& =\mathrm{e}^{\psi(x)} \frac{(v(x))^{\alpha}}{\sqrt{n !}} \frac{n !}{\alpha !} \\
& =\frac{\sqrt{n !}}{\alpha !} \mathrm{e}^{\psi(x)}(v(x))^{\alpha} .
\end{aligned}
$$

On the other hand, we have

$$
\left\langle E_{n, \alpha}, e_{x}\right\rangle=\int_{\mathbb{R}} \overline{E_{n, \alpha}(t)} \mathrm{e}^{i x t} \mu(\mathrm{d} t)=\int_{\mathbb{R}} \overline{E_{n, \alpha}(t)} \mathrm{e}^{i x t} \rho(t) \mathrm{d} t .
$$

This implies that

$$
\int_{\mathbb{R}} \rho(t) \overline{E_{n, \alpha}(t)} \mathrm{e}^{i x t} \mathrm{~d} t=\frac{\sqrt{n !}}{\alpha !} \mathrm{e}^{\psi(x)}(v(x))^{\alpha}
$$

or equivalently 


$$
\mathcal{F}\left(\rho \overline{E_{n, \alpha}}\right)(x)=\frac{\sqrt{n !}}{\alpha !} \mathrm{e}^{\psi(x)}(v(x))^{\alpha},
$$

where $\mathcal{F}$ denotes the Fourier transform on $L^{1}(v)$. Note that the function $\rho E_{n, \alpha}$ belongs to the space $L^{1}(v)$. It follows that

$$
\rho \overline{E_{n, \alpha}}=\mathcal{F}^{-1}\left(\frac{\sqrt{n !}}{\alpha !} \mathrm{e}^{\psi(\cdot)}(v(\cdot))^{\alpha}\right)
$$

which is equivalent to

$$
E_{n, \alpha}=\frac{\sqrt{n !}}{\alpha ! \rho} \overline{\mathcal{F}^{-1}\left(e^{\psi(\cdot)}(v(\cdot))^{\alpha}\right)}
$$

\section{Conclusion}

The infinite-divisibility of the distribution $\mu$ gives rise to the Kolmogorov isomorphism $U$, which was the principal bridge between the Fock space $\Gamma\left(L^{2}(v)\right)$ and $L^{2}(\mu)$ transforming, in such canonical way, the quantum decomposition identity to the tri-diagonal recurrence relation.

\section{Acknowledgements}

The authors gratefully acknowledge Qassim University, represented by the Deanship of Scientific Research, on the material support for this research under the number 3378 during the academic year $1436 \mathrm{AH} / 2015 \mathrm{AD}$.

\section{References}

[1] Hora, A. and Obata, N. (2007) Quantum Probability and Spectral Analysis of Graphs, Theoretical and Mathematical Physics. Springer, Berlin, Heidelberg.

[2] Asai, N., Kubo, I. and Kuo, H.H. (2003) Generating Functions of Orthogonal Polynomials and Szego-Jacobi Parameters. Probability and Mathematical Statistics, 23, 273-291.

[3] Accardi, L. and Bożejko, M. (1998) Interacting Fock Space and Gaussianization of Probability Measures. Infinite Dimensional Analysis, Quantum Probability and Related Topics, 1, 663-670. https://doi.org/10.1142/S0219025798000363

[4] Accardi, L., Rebei, H. and Riahi, A. (2013) The Quantum Decomposition of Infinitely Divisible Random Variables. Infinite Dimensional Analysis, Quantum Probability and Related Topics, 16, 1350012.

[5] Accardi, L., Rebei, H. and Riahi, A. (2014) The Quantum Decomposition Associated with the Lévy White Noise Processes without Moments. Probability and Mathematical Statistics, 34, 337-362.

[6] Accardi, L., Ouerdiane, H. and Rebei, H. (2010) On the Quadratic Heisenberg Group. Infinite Dimensional Analysis, Quantum Probability and Related Topics, 13, 551-587. https://doi.org/10.1142/S0219025710004231

[7] Rebei, H. (2015) The Generalized Heisenberg Group Arising from Weyl Relations, Quantum Studies. Mathematics and Foundations, 2, 323-350.

[8] Rebei, H. (2016) On the One Mode Quadratic Weyl Operators. Journal of Mathe- 
matical Analysis and Applications, 439, 135-153.

https://doi.org/10.1016/j.jmaa.2016.02.040

[9] Rebei, H., Al-Mohaimeed, B. and Riahi, A. (2015) Classical Versions of Quantum Stochastic Processes Associated with the Adapted Oscillator Algebra. International Journal of Innovation in Science and Mathematics, 3, 245-253.

[10] Accardi, L., Ouerdiane, H. and Rebei, H. (2012) Lévy Processes through Time Schift on Oscillator Weyl Algebra. Communications on Stochastic Analysis, 6, 125-155.

[11] Accardi, L., Ouerdiane, H. and Rebei, H. (2012) Renormalized Square of White Noise Quantum Time Shift. Communications on Stochastic Analysis, 6, 177-191.

[12] Accardi, L., Boukas, A. and Misiewicz, J. (2011) Existence of the Fock Representation for Current Algebras of the Galilei Algebra. QP-PQ: Quantum Probability and Related Topics, 27, 1-33.

[13] Sato, K.-I. (1999) Lévy Processes and Infinitely Divisible Distributions. Cambridge Stud. Adv. Math., Cambridge Univ. Press, Cambridge.

[14] Reed, M. and Simon, B. (1980) Functional Analysis, Methods of Modern Mathematical Physics. Vol. 1. Academic Press, INC., London.

[15] Stochel, J.B. (1992) Subnormality of Generalized Cration and Annihilation Operators on Bargmann's Space of Infinite Order. Integral Equations and Operator Theory, 15, 1011-1032. 